\title{
PAPR Reduction Performance by Active Constellation Extension for Diversity MIMO-OFDM Systems
}

\author{
Theodoros Tsiligkaridis and Douglas L. Jones \\ Department of Electrical and Computer Engineering, University of Illinois at Urbana-Champaign, Urbana, IL 61801, USA \\ Correspondence should be addressed to Theodoros Tsiligkaridis, tsiligk1@uiuc.edu
}

Received 6 June 2009; Accepted 28 September 2009

Academic Editor: Adam Panagos

Copyright (C 2010 T. Tsiligkaridis and D. L. Jones. This is an open access article distributed under the Creative Commons Attribution License, which permits unrestricted use, distribution, and reproduction in any medium, provided the original work is properly cited.

The V-BLAST wireless communication architecture, space-time block code (STBC), and space-frequency block code (SFBC) techniques are strong candidates for achieving very high data rates in $4 \mathrm{G}$ broadband wireless communications. This paper extends the efficient Active Constellation Extension Smart Gradient-Project (ACE-SGP) peak-to-average power (PAPR) reduction method to STBC, SFBC, and V-BLAST systems. Simulation results show 4.19 and $3.57 \mathrm{~dB}$ of PAPR reduction for the Alamouti STBC and SFBC, respectively.

\section{Introduction}

High peak power becomes a serious disadvantage when harnessing the power of MIMO-OFDM systems. As the $4 \mathrm{G}$ wireless revolution is demanding higher speeds and increased reliability, transmit diversity and spatial multiplexing techniques in MIMO systems are becoming more popular. Of these techniques, space-time block codes (STBCs), space-frequency block codes (SFBCs), and the Vertical Bell Labs Layered Space-Time (V-BLAST) architecture have gained wide use in many wireless standards, such as WiMAX, IEEE 802.11n, and 3GPP-LTE (3rd Generation Partnership Project-Long-Term Evolution). Space-time-frequency (STF) block coding schemes take advantage of diversity at a transmitting station often without requiring any channelstate information (CSI); at the same time, when using orthogonal block codes, they allow simple decoding at the receiver station. However, they still have the high peak-toaverage power ratio (PAPR) of OFDM, which requires high power amplifier backoff and results in low efficiency [1].

A robust peak power reduction technique called active constellation extension (ACE) reduces the PAPR of singleinput single-output (SISO) OFDM signals by extending the outer constellation points in a way that minimizes the PAPR of an OFDM transmit signal, without reducing the bandwidth or increasing bit-error-rate (BER) [1].
Recent work includes extensions of the concept of ACE using a modified smart gradient-project algorithm for MIMO-OFDM systems [2]. In this letter, we extend the efficient ACE-SGP method to STBC, SFBC, and V-BLAST OFDM systems. The proposed peak power reduction method can be applied to any STF block code, and its performance is bounded by the code structure.

\section{PAPR Properties of OFDM Signals}

OFDM modulation is performed by taking the inverse Discrete Fourier Transform (IDFT) of a block of $N$ QAMmodulated data symbols $X_{j}=\left[X_{0, j}, X_{1, j}, \ldots, X_{N-1, j}\right]^{T}$. The resulting baseband OFDM signal $x(t)$ can be expressed as

$$
x(t)=\sum_{k=0}^{N-1} X_{k} e^{j 2 \pi f_{n} t}, \quad 0 \leqslant t \leqslant N T .
$$

The analog PAPR of an OFDM symbol block can be computed as

$$
\operatorname{PAPR}(x(t))=\frac{\max _{0 \leqslant t \leqslant N T}|x(t)|^{2}}{E\left[|x(t)|^{2}\right]}=\frac{\max _{0 \leqslant t \leqslant N T}|x(t)|^{2}}{(1 / N T) \int_{0}^{N T}|x(t)|^{2} d t},
$$

where $f_{n}=n / N T$ and $T$ is the symbol period. 
Oversampling the discrete-time signal $x[n]$ to a factor of $L=4$ is sufficient for capturing the continuous-time peaks, as discussed in [3], and to prevent large analog "peak regrowth" at the D/A converter. To statistically evaluate the PAR performance, the complementary cumulative distribution function (CCDF) of the PAPR of the OFDM time signals is used to describe the probability of exceeding a given threshold $\gamma$, that is,

$$
\operatorname{CCDF}(\operatorname{PAPR}(x(t)))=\operatorname{Pr}[\operatorname{PAPR}(x(t))>\gamma] .
$$

\section{ACE-SGP STBC MIMO-OFDM Algorithm}

3.1. STBC MIMO-OFDM. The simplest $2 \times 2$ STBC with full diversity was first introduced by Alamouti in [4]. The encoder takes a block of two QAM symbols $X_{1}$ and $X_{2}$ and assigns it to the two transmit antennas according to the following code matrix:

$$
X_{A}=\left[\begin{array}{cc}
X_{1} & -X_{2}^{*} \\
X_{2} & X_{1}^{*}
\end{array}\right]^{T},
$$

where the $i$ th row represents the $i$ th transmission period and the $j$ th column corresponds to the $j$ th antenna's symbols.

In this letter, we also show simulation results for the following well-known orthogonal codes designed for four transmit antennas [5], with code rates $1 / 2$ and $3 / 4$, respectively:

$$
\begin{aligned}
X_{B} & =\left[\begin{array}{cccccccc}
X_{1} & -X_{2} & -X_{3} & -X_{4} & X_{1}^{*} & -X_{2}^{*} & -X_{3}^{*} & -X_{4}^{*} \\
X_{2} & X_{1} & X_{4} & -X_{3} & X_{2}^{*} & X_{1}^{*} & X_{4}^{*} & -X_{3}^{*} \\
X_{3} & -X_{4} & X_{1} & X_{2} & X_{3}^{*} & -X_{4}^{*} & X_{1}^{*} & X_{2}^{*} \\
X_{4} & X_{3} & -X_{2} & X_{1} & X_{4}^{*} & X_{3}^{*} & -X_{2}^{*} & X_{1}^{*}
\end{array}\right]^{T}, \\
X_{C} & =\left[\begin{array}{cccc}
X_{1} & 0 & X_{2} & -X_{3} \\
0 & X_{1} & X_{3}^{*} & X_{2}^{*} \\
-X_{2}^{*} & -X_{3} & X_{1}^{*} & 0 \\
X_{3}^{*} & -X_{2} & 0 & X_{1}^{*}
\end{array}\right]^{T} .
\end{aligned}
$$

3.2. STBC MIMO-OFDM Gradient-Project Algorithm. The solution to the PAPR reduction problem can be obtained with a fast gradient-project method, where only a couple of iterations are needed to considerably reduce the PAPR. A detailed discussion on the ACE-SGP algorithm can be found in [3].

Due to the structure of orthogonal space-time block codes, the SISO ACE-SGP algorithm need only be applied for the first transmission period, since it can be proven that complex sequences $X_{i}$ and $\pm X_{i}^{*}$ exhibit the same PAPR properties. Therefore, we only need to perform ACE-SGP processing on each SISO block independently, obtaining the minimum PAPR time-domain signal ready to transmit after adding the cyclic prefix for the first transmission period. To obtain the time-domain signals for the rest of the transmission periods, the scaling and conjugate DFT properties can be used to avoid the unnecessary IFFT operations for the remaining transmission periods across all transmit antennas:

$$
x^{*}\left[(-n)_{\bmod N}\right] \stackrel{\mathcal{F}}{\longleftrightarrow} X^{*}[k] .
$$

This property is especially useful for lower-rate codes, such as $X_{C}$.

\section{ACE-SGP SFBC MIMO-OFDM Algorithm}

4.1. SFBC MIMO-OFDM. Space-frequency block coding is another way to obtain diversity in order to improve the performance when employing multiple antennas at the transmitter and the receiver, particularly when there is frequency-selective fading. Coding occurs across adjacent subcarriers of the same OFDM symbol rather than on the same subcarrier of subsequent OFDM symbols (i.e., STBC), thus, reducing the transmission delay.

4.2. SFBC MIMO-OFDM Gradient-Project Algorithm. The main idea of the ACE-SGP algorithm is to look at the residual clipped-off signal and project it onto the space of allowable extension vectors. The result is a peak-reduction signal scaled by an optimal step size subtracted from the timedomain signal. For STF block codes, to correctly decode the signal, we project the residual clipped-off signal into the space of allowable STFBC extensions, which depends on the code being used.

In general, the set of possible ACE vectors will be convex. The three convex sets are the following:

(1) the set $S_{A}$, consisting of all vectors $\mathbf{y} \in \mathbb{R}^{N}$ such that $|\mathbf{y}|_{\infty} \leqslant A$ for some positive constant $A$,

(2) the set $S_{C}$, an $N$-dimensional subspace of $\mathbb{R}^{N}$, consisting of all vectors $\mathbf{y}$ with FFT $\mathbf{Y}$ that satisfy the data-dependent ACE restrictions,

(3) the set $S_{B}$, a $K$-dimensional $\left(K=M_{T} p\right.$, where $p$ is the number of transmission periods) subspace of $\mathbb{R}^{N}$, consisting of all vectors $\mathbf{x}$ that yield the largest allowable ACE extension while maintaining the STFBC linear decoding properties.

Below is a basic pseudocode for an STFBC OFDM algorithm based on the gradient-project method.

(1) Starting with the input bit stream, break it up into blocks of size $N$ for parallel transmission, where there are $N$ subchannels total. Consider transmission of $p$ OFDM blocks per antenna. Perform M-QAM modulation to obtain the frequency-domain symbols, $X_{k}$.

(2) Add transmit diversity by performing space-time block coding using an STFBC and determine the allowable extensions for each subchannel.

(3) Take the IFFT for each antenna's signal to construct the time signal, $x^{0}[n, l]_{t}$, for each antenna $l$ ( $n$ is the subchannel index, $l$ is the antenna index, and $t$ is the transmission period index). Set $i=0$. 
(4) Clip any $\left|x^{i}[n, l]_{t}\right| \geqslant A$ in magnitude for some clip level $A$ (i.e., project onto $S_{A}$ ) to obtain

$$
\bar{x}[n, l]_{t}= \begin{cases}x^{i}[n, l]_{t}, & \left|x^{i}[n, l]_{t}\right| \leqslant A, \\ A e^{j \theta[n, l]_{t}}, & \left|x^{i}[n, l]_{t}\right|>A,\end{cases}
$$

where

$$
x^{i}[n]_{t}=\left|x^{i}[n, l]_{t}\right| e^{j \theta[n, l]_{t}}
$$

(5) Compute the clipped signal portion (nonzero only at clipped samples):

$$
c_{\text {clip }}[n, l]_{t}=\bar{x}[n, l]_{t}-x^{i}[n, l]_{t} .
$$

(6) Apply an FFT to each antenna's clipped signal to obtain $C_{\text {clip }}[k, l]_{t}$.

(7) Keep only the components of $C_{\text {clip }}[k, l]_{t}$ which are acceptable extension directions for the given subchannel constellations and set all remaining elements to zero (i.e., project onto $S_{C}$ ).

(8) Apply the STFBC constraint (i.e., project onto $S_{B}$ ) by taking the maximum ACE extension and enforcing it on each block according to the code matrix. (Note that this step ensures that the STFBC constraints are still satisfied; thus the optimal decoding methods associated with the STFBC are still optimal. For example, in the case of orthogonal SFBC, linear maximum-likelihood decoding is still optimal.)

(9) Apply an IFFT to obtain $c[n, l]_{t}$.

(10) Determine a step size $\mu[l]_{t}$ according to the smart gradient criterion (i.e., for quick convergence, see [3]):

$$
x^{i+1}[n, l]_{t}=x^{i}[n, l]_{t}+\mu[l]_{t} c[n, l]_{t} .
$$

(11) If an acceptable PAPR or a maximum iteration count has not been reached, update $i=i+1$ and return to Step 4. Otherwise, stop PAPR reduction.

This algorithm's complexity is $O\left(M_{T} N L \log N L\right.$ ) (for each iteration) due to the oversampled IFFT/FFT operations. The algorithm converges sufficiently after 3 to 4 iterations. The algorithm described here can be applied to a general STF block code and it greatly simplifies for orthogonal STBC/SFBC's (i.e., as in Section 3).

\section{ACE-SGP V-BLAST MIMO-OFDM Algorithm}

Unlike STBCs, V-BLAST has a multiplexing gain but lacks a diversity gain [6]. Because independent data streams are sent on the different transmit antennas, the ACE-SGP algorithm can be applied on each antennas time signal independently, like in the SISO case described in great detail in [3].

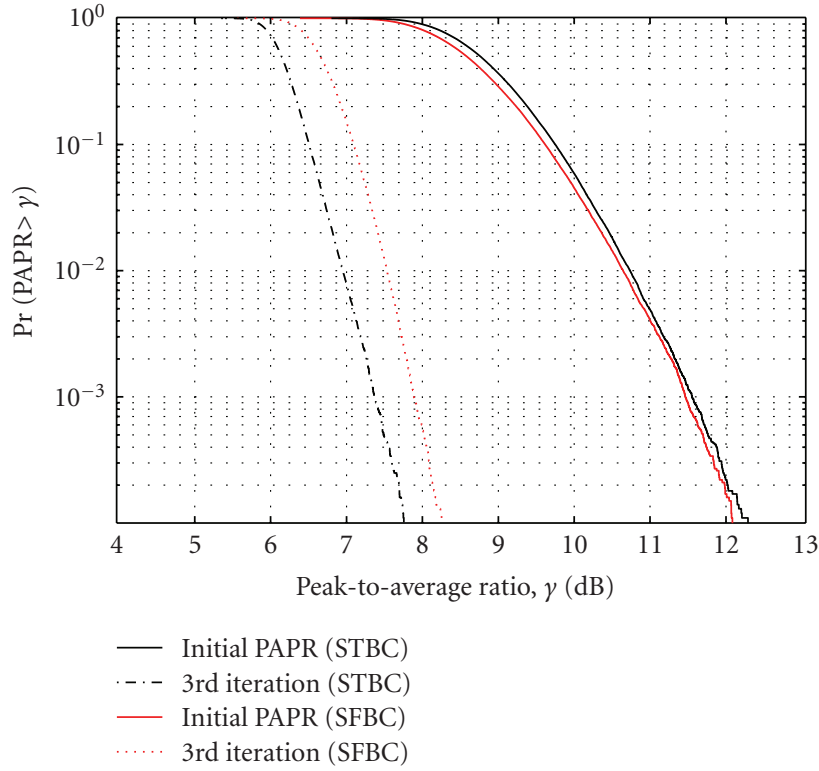

Figure 1: CCDF Results with 2-Tx antenna STBC/SFBC using 256 subcarriers employing QPSK.

TABle 1: PAPR Reduction results for Different Space-Time Block Codes and Modulation Types.

\begin{tabular}{lcccc}
\hline STBC & $\Delta P_{4}(\mathrm{~dB})$ & $\Delta P_{16}(\mathrm{~dB})$ & $\Delta P_{64}(\mathrm{~dB})$ & $P^{0}(\mathrm{~dB})$ \\
\hline$X_{A}$ & 4.19 & 2.61 & 1.46 & 11.55 \\
$X_{B}$ & 3.96 & 2.30 & 1.08 & 11.74 \\
$X_{C}$ & 2.32 & 0.78 & 0.20 & 12.85 \\
\hline
\end{tabular}

TABLe 2: PAPR Reduction results for Different Space-Frequency Block Codes and Modulation Types.

\begin{tabular}{lcccc}
\hline SFBC & $\Delta P_{4}(\mathrm{~dB})$ & $\Delta P_{16}(\mathrm{~dB})$ & $\Delta P_{64}(\mathrm{~dB})$ & $P^{0}(\mathrm{~dB})$ \\
\hline$X_{A}$ & $3.57 \mathrm{~dB}$ & $2.52 \mathrm{~dB}$ & $1.65 \mathrm{~dB}$ & $11.46 \mathrm{~dB}$ \\
$X_{B}$ & $2.27 \mathrm{~dB}$ & $1.60 \mathrm{~dB}$ & $1.28 \mathrm{~dB}$ & $11.80 \mathrm{~dB}$ \\
$X_{C}$ & $2.28 \mathrm{~dB}$ & $0.88 \mathrm{~dB}$ & $0.52 \mathrm{~dB}$ & $11.57 \mathrm{~dB}$ \\
\hline
\end{tabular}

\section{Simulation Results}

Results of the ACE-SGP algorithm for STBC and SFBC MIMO-OFDM are shown in Figure 1. The algorithm was tested for a complex-baseband STBC OFDM two-antenna system with $N=256$ subchannels employing QPSK using $10^{5}$ randomly generated OFDM symbol blocks. A level $A$ of $4.0 \mathrm{~dB}$ above the average power was used to obtain the clipped signal to project onto the space of allowable extension vectors, and an upsampling factor of $L=4$ was used to approximate analog PAPR reduction. Failure to process oversampled digital signals leads to large peak regrowth at the $\mathrm{D} / \mathrm{A}$ converter. Oversampling was performed by frequency-domain zero-padding and using oversampled IFFT operations. 
TABLE 3: PAPR Reduction results for V-BLAST under Different Number of Antennas and Modulation Types.

\begin{tabular}{lcccc}
\hline$M_{T}$ & $\Delta P_{4}(\mathrm{~dB})$ & $\Delta P_{16}(\mathrm{~dB})$ & $\Delta P_{64}(\mathrm{~dB})$ & $P^{0}(\mathrm{~dB})$ \\
\hline 2 & $4.04 \mathrm{~dB}$ & $2.50 \mathrm{~dB}$ & $1.27 \mathrm{~dB}$ & $11.52 \mathrm{~dB}$ \\
4 & $4.44 \mathrm{~dB}$ & $3.04 \mathrm{~dB}$ & $1.88 \mathrm{~dB}$ & $11.69 \mathrm{~dB}$ \\
\hline
\end{tabular}

As shown in Figure 1, for Alamouti STBC, looking at a $10^{-3}$ symbol clip probability level, the third iteration achieves a PAPR reduction of approximately $4.19 \mathrm{~dB}$. Further iterations provide negligible performance increases. Similarly, for Alamouti SFBC, a PAPR reduction of $3.57 \mathrm{~dB}$ is achieved in the third iteration. Due to the constellation extension, the average power of the transmit signal increases by $0.86 \mathrm{~dB}$ and by $1.08 \mathrm{~dB}$ (for each of the two antennas) under Alamouti STBC and SFBC, respectively. (The average power before any ACE processing is used in the PAPR calculation to avoid biasing of the results with the newly increased average power.)

Tables 1 and 2 summarize the PAPR reduction gains obtained using a 256-subcarrier MIMO-OFDM system when looking at a $10^{-3}$ symbol clip probability. (In Tables 1, 2, and $3, \Delta P_{4}$ denotes the reduction in PAPR for 4-QAM, $\Delta P_{16}$ denotes the reduction in PAPR for 16-QAM, $\Delta P_{64}$ denotes the reduction in PAPR for 64-QAM, and $P^{0}$ denotes the initial PAPR) An upsampling factor $L=4$ and the clipping levels, $A$, used were $4.0,5.0$, and $5.0 \mathrm{~dB}$ above the average power, for codes $X_{A}, X_{B}$, and $X_{C}$, respectively.

Table 3 summarizes the PAPR reduction gains obtained for V-BLAST, which are all obtained for a 256-subcarrier MIMO-OFDM system when looking at a $10^{-3}$ symbol clip probability. A clipping levels $A$ of 4.0 and $6.0 \mathrm{~dB}$ above the average power were used for two and four transmit antennas, respectively (with $L=8$ ). Larger PAPR reduction gains were obtained for the V-BLAST MIMO-OFDM systems than the STBC/SFBC cases because there is no constraint across the signals being sent out from the antennas (i.e., independent streams).

Figure 2 evaluates the BER performance of a $2 \times 2$ STBC MIMO-OFDM system under QPSK modulation and ACE-SGP processing with and without amplifier saturation, assuming additive white Gaussian noise (AWGN) conditions. The nonlinear high-power amplifier model used was based on [7], with companding parameter of 6, saturation output amplitude corresponding to single-carrier QPSK, and backoffs of 0 and $3 \mathrm{~dB}$. The backoff was implemented by raising the saturation amplitude level. The required SNR to achieve a BER of $10^{-2}$ is $2.2 \mathrm{~dB}$ less with ACE (versus without ACE) for $0 \mathrm{~dB}$ backoff and $1.0 \mathrm{~dB}$ less for a $3 \mathrm{~dB}$ backoff. Even without saturation, ACE slightly improves the BER due to increased margins on the extended symbols.

\section{Conclusions}

An extended method of ACE reduces the PAPR for spacetime-frequency block-coded MIMO-OFDM systems without compromising BER performance. Simulations showed that a PAPR reduction gain of 4.19 and $3.57 \mathrm{~dB}$ can be

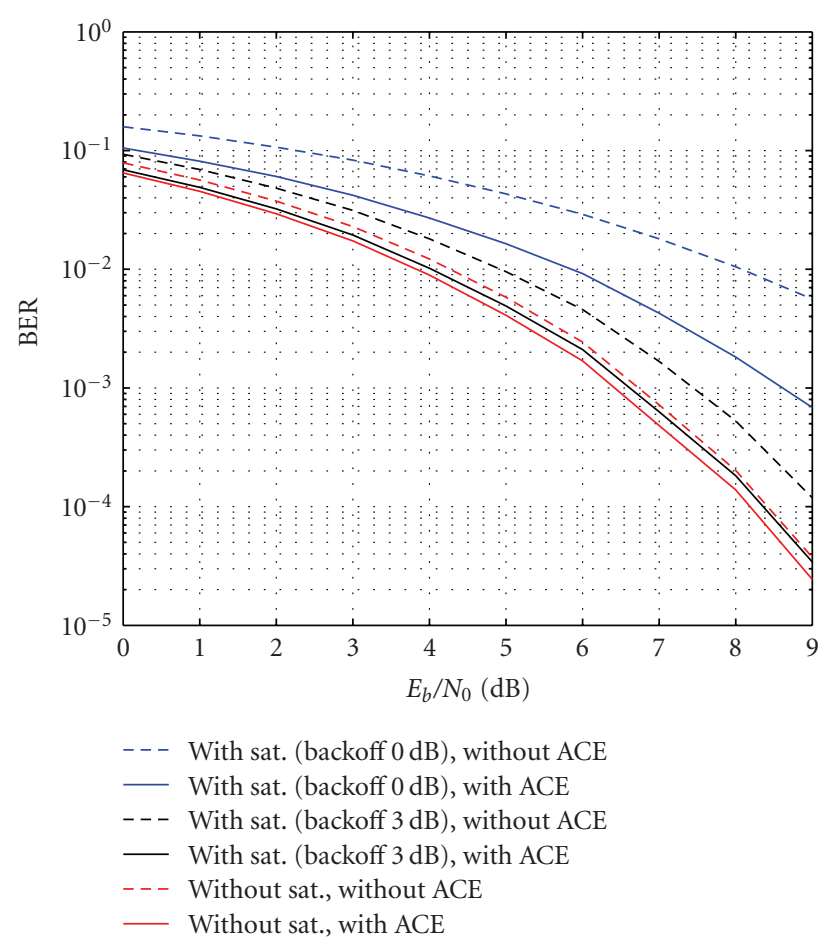

FIgure 2: BER Performance Results with 2-Tx antenna STBC using 256 subcarriers employing QPSK under AWGN conditions.

obtained under QPSK for Alamouti STBC and SFBC, respectively. On the other hand, gains above $4 \mathrm{~dB}$ were obtained for two and four antennas under QPSK for V-BLAST. Results show that as the number of antennas increases, the block code constraint reduces the ACE PAPR reduction gain for space-time-frequency-coded MIMO-OFDM systems, due to the reduced number of degrees of freedom caused by structured redundancy. However, the ACE PAPR reduction gain increases with the number of antennas for uncoded MIMOOFDM systems (i.e., V-BLAST). The PAPR reduction gains obtained are larger than those observed for the SISO case [3], and they seem most promising for small constellations (i.e., QPSK and 16-QAM).

\section{References}

[1] D. L. Jones, "Peak power reduction in OFDM and DMT via active channel modification," in Proceedings of the Asilomar Conference on Signals, Systems, and Computers, vol. 2, pp. 10761079, Piscataway, NJ, USA, 1999.

[2] B. S. Krongold, G. R. Woo, and D. L. Jones, "Fast active constellation extension for MIMO-OFDM PAR reduction," in Proceedings of the IEEE International Conference on Communications (ICC '05), pp. 1476-1479, Pacific Groove, Calif, USA, October-November 2005.

[3] B. S. Krongold and D. L. Jones, "PAR reduction in OFDM via active constellation extension," IEEE Transactions on Broadcasting, vol. 49, no. 3, pp. 258-268, 2003.

[4] S. M. Alamouti, "A simple transmit diversity technique for wireless communications," IEEE Journal on Selected Areas in Communications, vol. 16, no. 8, pp. 1451-1458, 1998. 
[5] V. Tarokh, H. Jafarkhani, and A. R. Calderbank, "Space-time block codes from orthogonal designs," IEEE Transactions on Information Theory, vol. 45, no. 5, pp. 1456-1467, 1999.

[6] P. W. Wolniansky, G. J. Foschini, G. D. Golden, and R. A. Valenzuela, "V-BLAST: an architecture for realizing very high data rates over the rich-scattering wireless channel," in Proceedings of the International Symposium on Signals, Systems and Electronics (ISSSE '98), pp. 295-300, Pisa, Italy, October 1998.

[7] T. G. Pratt, N. Jones, L. Smee, and M. Torrey, "OFDM link performance with companding for PAPR reduction in the presence of non-linear amplification," IEEE Transactions on Broadcasting, vol. 52, no. 2, pp. 261-267, 2006. 

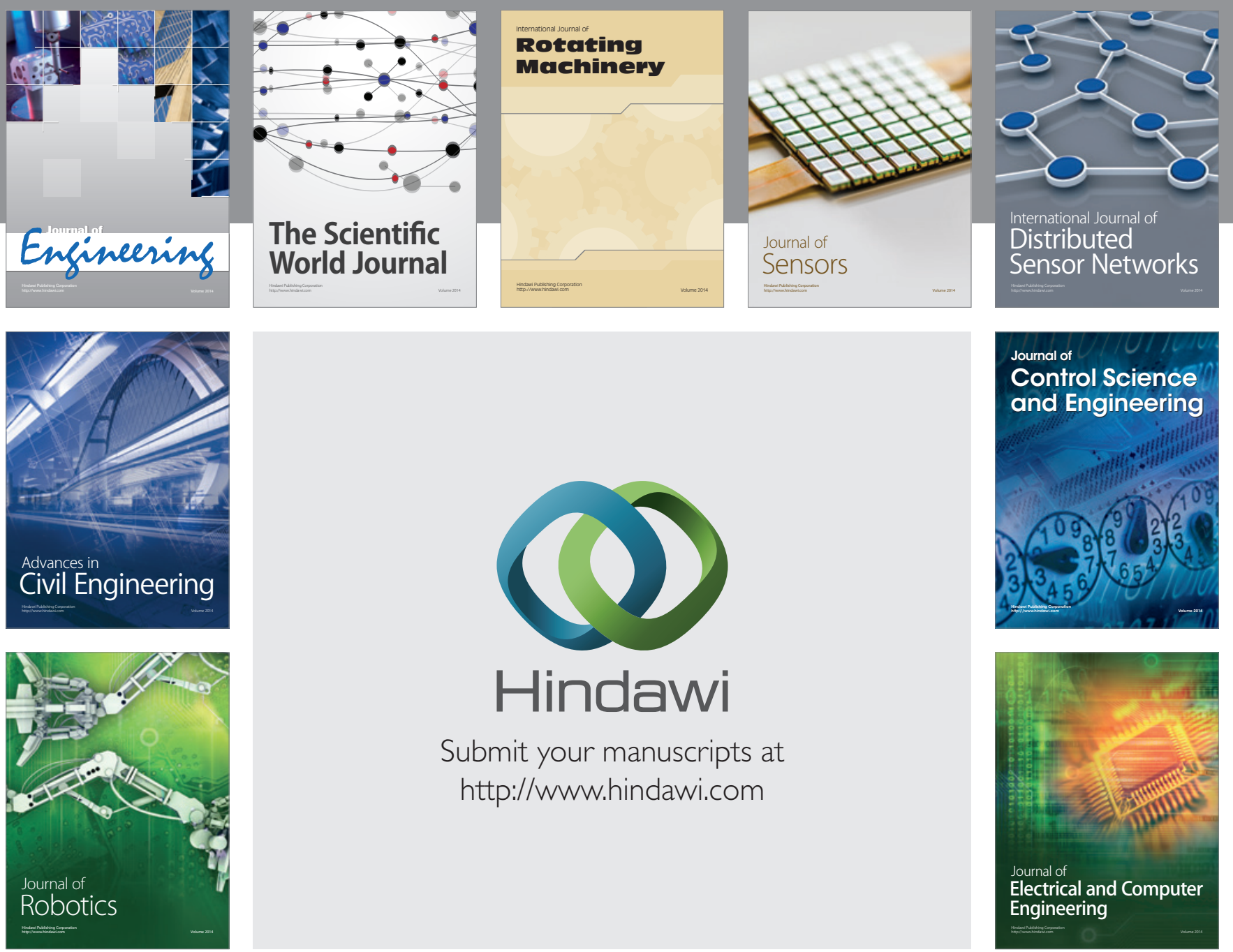

Submit your manuscripts at

http://www.hindawi.com
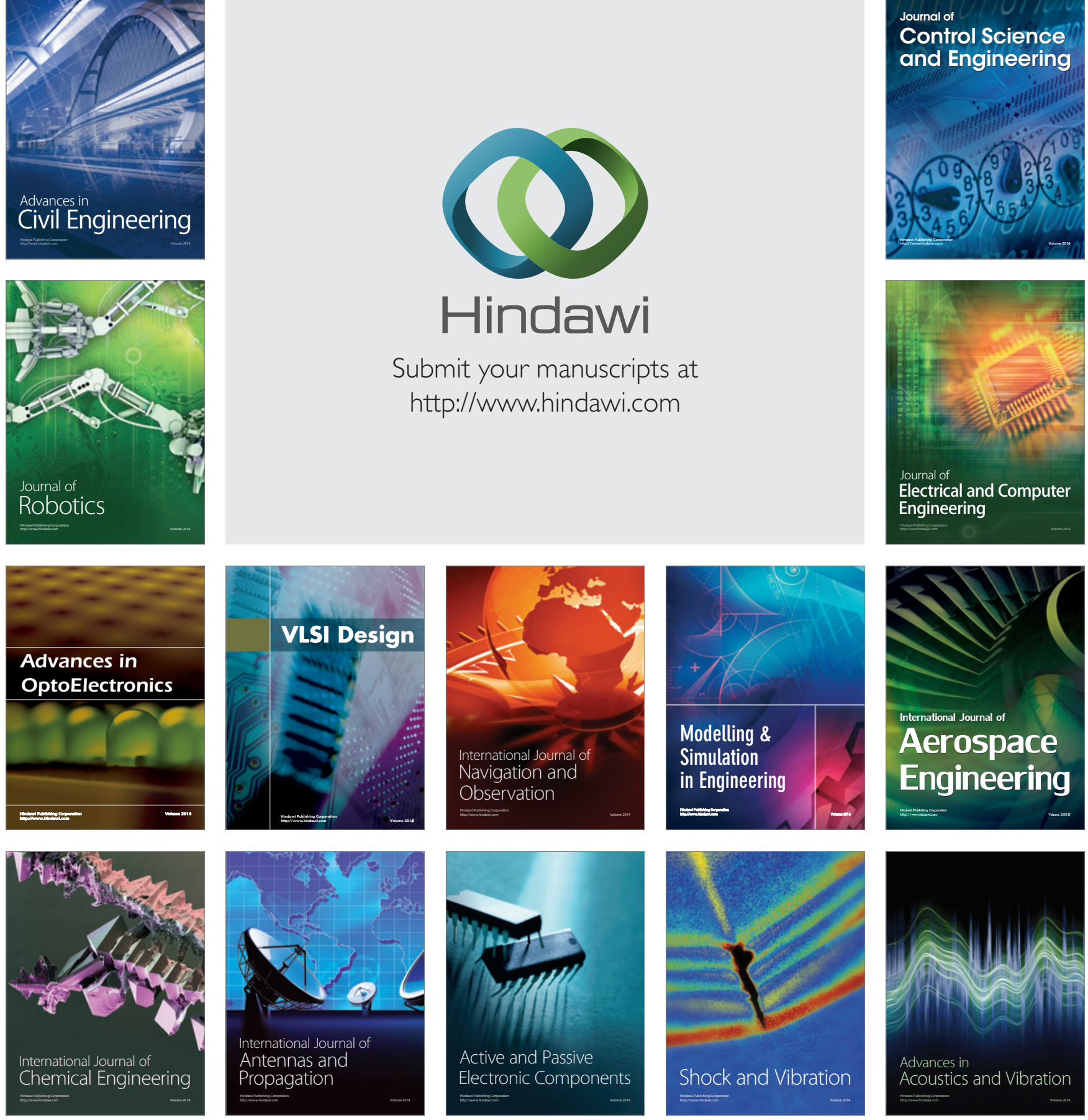